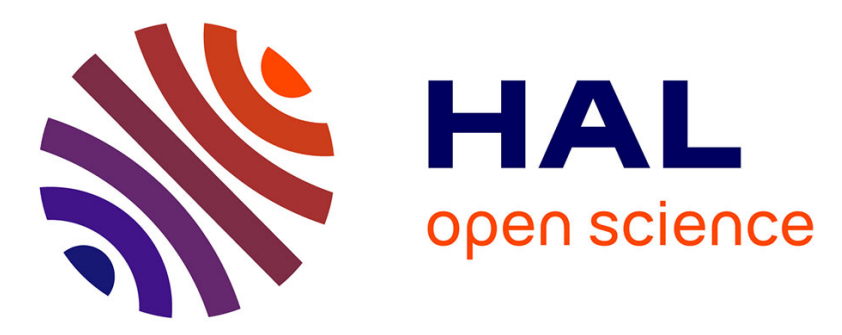

\title{
Etude comparative de quelques cultivars de blé, seigle et triticale dans le Nord du Portugal. II. Adaptation du matériel végétal au milieu
}

Michel Bernard, Henrique Guedes Pinto

\section{- To cite this version:}

Michel Bernard, Henrique Guedes Pinto. Etude comparative de quelques cultivars de blé, seigle et triticale dans le Nord du Portugal. II. Adaptation du matériel végétal au milieu. Agronomie, 1983, 3 (8), pp.723-733. hal-00884565

\section{HAL Id: hal-00884565 https://hal.science/hal-00884565}

Submitted on 1 Jan 1983

HAL is a multi-disciplinary open access archive for the deposit and dissemination of scientific research documents, whether they are published or not. The documents may come from teaching and research institutions in France or abroad, or from public or private research centers.
L'archive ouverte pluridisciplinaire HAL, est destinée au dépôt et à la diffusion de documents scientifiques de niveau recherche, publiés ou non, émanant des établissements d'enseignement et de recherche français ou étrangers, des laboratoires publics ou privés. 


\title{
Etude comparative de quelques cultivars de blé, seigle et triticale dans le Nord du Portugal. II. Adaptation du matériel végétal au milieu
}

\author{
Michel BERNARD \& Henrique GUEDES PINTO * \\ * I.N.R.A. Station d'Amélioration des Plantes, F 63000 Clermont-Ferrand \\ * Instituto Universitario de Tras-os-Montes e Alto Douro, Vila Real, Portugal
}

\begin{abstract}
A la suite d'une expérimentation multilocale réalisée en 1978 dans la province de Tras-Os-Montes (Portugal), comprenant 17 génotypes de blé et triticale d'origines différentes et 4 populations locales de seigle, on a cherché à préciser le degré d'adaptation de chaque génotype ou population aux différents environnements. On a dans ce but utilisé 2 techniques d'analyse statistique : le modèle de MANDEL de décomposition de la variance s'appliquant aux essais 2 voies sans répétition et l'analyse en composantes principales. Dans les 2 cas, les génotypes ont été caractérisés par leurs productions de grain, de paille, de protéines, leurs teneurs en protéines et leurs poids de 1000 grains dans les différents lieux.

L'analyse selon MANDEL dégage, pour chaque génotype, l'effet principal et le paramètre de participation à l'interaction génotype-milieu, ceci pour chaque caractère mesuré. Ces 2 paramètres définissent le potentiel moyen d'un génotype dans la région considérée et son potentiel d'adaptation spécifique aux différents environnements.

Les groupes présentant des valeurs convenables de potentiel moyen sont les seigles locaux et le blé «Barbela», d'une part, les génotypes INRA de triticale, d'autre part. Chaque groupe est cependant étroitement adapté à des environnements différents : milieux pauvres et relativement froids pour le premier, milieux riches et assez chauds pour le second.

Dans les 2 types d'analyse, les génotypes de triticale INRA et CIMMYT (du type « Armadillo ») apparaissent en opposition. "Beagle ", triticale CIMMYT non substitué, se trouve en situation intermédiaire entre les 2 groupes.

L'intérêt de la définition du milieu, préliminaire à un programme de sélection, est discuté.
\end{abstract}

Mots clés additionnels : Analyse multilocale, interaction génotype $\times$ milieu, rendement, poids de 1000 grains, teneur en protéines.

Comparative study of some cultivars of wheat, rye and triticale in Northern Portugal. II. Adaptation to the environment.

Regional yield trials involving 3 cultivars of common wheat, 14 genotypes of hexaploid triticale (from both INRA and CIMMYT) and 4 local populations of rye were carried out in 1978 in the North-Eastern province of Tras-os-Montes (Portugal). For each genotype we tried to specify its adaptation level to the different environments. Two statistical analyses were used : first, an analysis of variance and partioning of interaction according to MANDEL's model, and second a principal component analysis. In both cases, the genotypes were defined by : their production of grain, straw and protein, their protein content and their 1.000 kernel weight at the different locations.

The analysis according to MANDEL separated, for each genotype, a main effect and a parameter for participation in the genotype $\times$ environment interaction. The main effect defines the average production potential of the genotype considered for the growing area; the participation parameter expresses its potential for specific adaptation to the different environments.

The best mean production potentials were obtained for the local population of rye, the wheat variety "Barbela" and the INRA triticale genotypes.

However these groups were not adapted to the same environments : poor soils and low temperature for the ryes and "Barbela", favourable soil conditions and higher temperatures for the INRA triticale.

In the 2 types of analysis, the INRA and CIMMYT (" Armadillo" family) triticale genotypes were in contrast. "Beagle", a non-substituted CIMMYT triticale, was in an intermediate position between the 2 groups.

The value of defining the environment, prior to a breeding program, is discussed.

Additional key words: Multilocation analysis, genotype - environment interaction, yield, 1.000 kernel weight, protein content. 


\section{INTRODUCTION}

Il relève du domaine de l'amélioration des plantes non seulement de savoir créer des génotypes productifs et intéressants à exploiter dans un millieu donné, mais aussi de connaître les facultés d'adaptation de ces génotypes à une gamme de milieux. Ces réactions d'adaptation font appel à des mécanismes physiologiques, mais leur amplitude et leur sens sont évidemment contrôlés par le génotype. La connaissance de ces réponses doit permettre de faire des choix pertinents tant au niveau de la commercialisation de variétés qu'à celui des géniteurs entrant dans un croisement. On peut en effet considérer la « souplesse d'adaptation » d'un génotype comme une caractéristique présentant le même intérêt qu'une résistance à une maladie, étant d'ailleurs entendu que ces 2 types de caractère ne sont probablement pas antagonistes.

Dans un article précédent (GUEDES-PINTO \& BERNARD, 1983), nous avons étudié les productions en grain, en paille, en protéines, ainsi que le poids de 1000 grains et les teneurs en protéines, de 17 génotypes de blé tendre et triticale et 4 populations de seigle, dans 5 localités représentatives des milieux de la province Nord du Portugal, en 1978. Nous avons ainsi défini les génotypes ou populations qui sont, en moyenne, les plus intéressants pour la gamme de milieux considérés et tenté de préciser quels sont ceux qui paraissent le mieux adaptés à tel ou tel type de milieu.

Afin d'avoir une vision plus précise des différences de comportement entre les différents groupes de génotypes et, si possible, entre génotypes d'un même groupe, nous avons cherché, d'une part, à analyser les interactions génotype $\times$ milieu pour les différents caractères mesurés grâce au modèle de MANDEL (1971) présenté par LEFORT (1977) et BRIAN (1978), d'autre part, à visualiser les comportements grâce à la technique d'analyse en composantes principales (ACP) sur l'ensemble des données, dans un système d'axes défini par les 5 paramètres de production mesurés dans les différents envirorınements.

\section{MATÉRIEL ET MÉTHODES}

\section{A. Le matériel}

Il a été présenté dans l'article précédent. Rappelons qu'il se compose de :

- 3 cultivars de blé ;

- 4 populations locales de seigle ;

- 7 génotypes de triticale INRA;

- 7 génotypes de triticale CIMMYT.

L'ensemble de ce matériel a été expérimenté dans 5 lieux différant tant sur le plan pédologique que sur le plan climatique et, particulièrement, au niveau du régime des températures dans la zone Nord du Portugal.

Les paramètres mesurés en chaque lieu sur chaque répétition ont été :

- la production de grain ;

- la production de paille.

Les paramètres mesurés pour chaque génotype en chaque lieu sur le mélange des répétitions ont été :

- le poids de 1000 grains ;

- la teneur en protéines du grain en p. 100 de la matière sèche.

La production de protéines a été évaluée en multipliant la production de grain par la teneur en protéines.
B. Analyse des productions selon le modèle de MANDEL (1971)

Pour une production $\mathrm{Y}_{\mathrm{ij}}$ mesurée pour le génotype $\mathrm{i}$ dans le milieu $j$, le modèle général s'écrit:

$$
Y_{i j}=\mu+\alpha_{i}+B j+\gamma_{i j}+\varepsilon_{i j}
$$

où $\mu$ est estimé par la moyenne générale des données. $\alpha_{i}$ et $\beta_{\mathrm{j}}$ représentent respectivement les effets ligne (« génotypes») et colonne ( « lieux»), $\gamma_{\mathrm{ij}}$ représentant l'interaction (ligne $\times$ colonne) et $\varepsilon_{i j}$ la variation aléatoire.

L'intérêt de l'analyse de MANDEL est de permettre de structurer aussi complètement que possible le terme « interaction ", en considérant que les paramètres d'interaction sont composés d'une somme de termes multiplicatifs. Le modèle complet s'écrit alors :

$$
\begin{aligned}
& Y_{i j}=\mu+L_{1 i}+C c_{j}+\underbrace{\Theta_{1} u_{1 i} v_{1 j}+\Theta_{2} u_{2 i} v_{2 j}+\ldots} \\
& \left(\alpha_{\mathrm{i}}\right) \\
& \left(\gamma_{\mathrm{ij}}\right)
\end{aligned}
$$

Les paramètres $l_{i}$ et $c_{j}$ affectés des coefficients respectifs $L$ et $\mathrm{C}$, représentent les effets lignes et colonnes et sont cstimés classiquement par la méthode des moindres carrés. Les différents termes multiplicatifs $\Theta \mathrm{u}_{\mathrm{i}} \mathrm{v}_{\mathrm{j}}$ rendent compte tour à tour d'une partie de la somme des carrés des résidus $\mathrm{d}_{\mathrm{ij}}$ :

$$
\mathrm{d}_{\mathrm{ij}}=\mathrm{Y}_{\mathrm{ij}}-\mathrm{Y}_{\mathrm{i} \cdot}-\mathrm{Y}_{\cdot \mathrm{j}}+\mathrm{Y}_{.}
$$

La signification de l'interaction est établie par le test $U$ de JOHNSON \& GRAYBILL (1972), calculé comme suit :

$$
\mathrm{U}=\frac{\mathrm{SCE}\left(\Theta_{1} \mathrm{u}_{1 \mathrm{i}} \mathrm{v}_{\mathrm{ij}}\right)}{\operatorname{SCE} \ll \text { Interaction } »}
$$

U s'exprime en p. 100 .

\section{Analyse en composantes principales}

Les caractères ont été étudiés globalement dans une analyse en composantes principales (ANDERSON, 1960 ; BENZECRI, 1973). Ce type d'analyse, qui se rattache à l'analyse discriminante, permet de visualiser les positions relatives des 21 génotypes étudiés dans un système d'axes défini à partir des valeurs obtenues pour chacun d'eux par les 5 caractères (PG : production de grain ; $\mathrm{PA}$ : production de paille; TP : teneur en protéines; PP : production de protéines ; MG : poids de 1000 grains) dans les 5 lieux (Vila Real : TT2 ; Vila Real, TT3 ; Carlão : C ; Montalegre : M ; Bragança : B). Il permet en outre de dégager les «tendances » de comportement des génotypes vis-à-vis des caractères entrant dans l'analyse.

Les calculs ont été effectués sur l'ordinateur du Centre de Calcul Scientifique de l'«Instituto Gulbenkian de Ciencias », Oeiras, Portugal, à partir d'un programme mis au point à la Station d'Amélioration des Plantes INRA de Clermont-Ferrand.

\section{RÉSULTATS}

\section{A. Analyse de Mandel}

Pour les 5 caractères étudiés, le tableau 1 présente les valeurs obtenues pour les paramètres $\mu, L$ et $C$, les rapports $F$ des variances testant les effets « génotypes » et « milieu », les valeurs de $U$ testant l'interaction. 
TABLEAU 1

Valeurs obtenues pour les paramètres $\mu, L$ et $C$ dans l'analyse de MANDEL pour les différents caractères étudiés. Values of the parameters $\mu, L$ and $C$ in the MANDEL analysis for the different characteristics.

\begin{tabular}{|c|c|c|c|c|c|c|}
\hline & $\mu$ & $\begin{array}{c}\mathrm{L} \\
\text { (génotypes) }\end{array}$ & $\begin{array}{c}\mathrm{C} \\
\text { (milieux) }\end{array}$ & $\mathrm{U}\left({ }^{1}\right)$ & $\underset{\text { \& milieux } »}{ }$ & $\begin{array}{c}F \\
\text { «génotypes » }\end{array}$ \\
\hline Production grain $(q / h a)$ & 21,1 & 50,48 & 20,42 & $84,31 * *$ & $24,88 * *$ & $7,24 * *$ \\
\hline Production paille $(\mathrm{q} / \mathrm{ha})$ & 65,65 & 67,05 & 83,04 & $84,35 * *$ & $101,75 * *$ & $7,24 * *$ \\
\hline PMG (g) & 40,65 & 32,52 & 8,46 & $66,35 * *$ & $29,74 * *$ & $20,94 * *$ \\
\hline TP (\% MS) & 10,62 & 10,95 & 2,43 & $56,72 * *$ & $36,43 * *$ & $35,18 * *$ \\
\hline Production protéines $(\mathrm{kg} / \mathrm{ha})$ & 206,6 & 372,2 & 243,9 & $87,2 * *$ & $45,86 * *$ & $5,08 * *$ \\
\hline
\end{tabular}

( ${ }^{1}$ ) Exprimé en p. 100 de la SCE «Interaction».

TABLEAU 2

Valeurs des effets principaux $l_{i}$ et des participations $u_{i}$ (au terme multiplicatif de rang 1 structurant l'interaction) des génotypes pour les 5 parametres étudiés (analyse selon MANDEL).

Values of the main genotype effects $l_{i}$ and of the genotype participation $u_{i}$ (to the first term of the interaction) for the 5 parameters studied.

\begin{tabular}{|c|c|c|c|c|c|c|c|c|c|c|c|}
\hline & & \multicolumn{2}{|c|}{ Produc. Grains } & \multicolumn{2}{|c|}{ Produc. Paille } & \multicolumn{2}{|c|}{ Pds 1000 grains } & \multicolumn{2}{|c|}{ Teneur protéines } & \multicolumn{2}{|c|}{ Produc. protéines } \\
\hline & & $\begin{array}{c}\text { Effet } \\
\text { du } \\
\text { génotype }\end{array}$ & $\begin{array}{l}\text { Val. inte. } \\
\text { rac. } \mathrm{G} \times \mathrm{E} \\
\mathrm{du} \\
\text { génotype }\end{array}$ & $\begin{array}{c}\text { Effet } \\
\text { du } \\
\text { génotype }\end{array}$ & $\begin{array}{l}\text { Val. inte. } \\
\text { rac. } \mathrm{G} \times \mathrm{E} \\
\mathrm{du} \\
\text { génotype }\end{array}$ & $\begin{array}{c}\text { Effet } \\
\text { du } \\
\text { génotype }\end{array}$ & $\begin{array}{l}\text { Val. inte } \\
\text { rac. } G \times E \\
\text { du } \\
\text { génotype }\end{array}$ & $\begin{array}{c}\text { Effet } \\
\text { du } \\
\text { génotype }\end{array}$ & $\begin{array}{l}\text { Val. inte. } \\
\text { rac. } \mathrm{G} \times \mathrm{E} \\
\text { du } \\
\text { génotype }\end{array}$ & $\begin{array}{c}\text { Effet } \\
\text { du } \\
\text { génotype }\end{array}$ & $\begin{array}{l}\text { Val. inte. } \\
\text { rac. } \mathrm{G} \times \mathrm{E} \\
\text { du } \\
\text { génotype }\end{array}$ \\
\hline$\frac{0}{m}$ & $\begin{array}{l}\text { Barbela } \\
\text { Autonomia } \\
7 \text { Cerros }\end{array}$ & $\begin{array}{l}-0,01 \\
-0,24 \\
-0,22\end{array}$ & $\begin{array}{l}-0,14 \\
-0,18 \\
-0,02\end{array}$ & $\begin{array}{l}-0,001 \\
-0,41 \\
-0,37\end{array}$ & $\begin{array}{r}0,07 \\
-0,08 \\
0,10\end{array}$ & $\begin{array}{r}0,34 \\
-0,12 \\
-0,29\end{array}$ & $\begin{array}{c}-0.38 \\
0,02 \\
0,04\end{array}$ & $\begin{array}{l}-0,12 \\
-0,05 \\
-0,10\end{array}$ & $\begin{array}{r}-0,08 \\
-0,34 \\
0,02\end{array}$ & $\begin{array}{l}-0,03 \\
-0,32 \\
-0,28\end{array}$ & $\begin{array}{l}-0,14 \\
-0,27 \\
-0,06\end{array}$ \\
\hline$\frac{0}{.0}$ & $\begin{array}{l}\text { Reg Vila Pouca } \\
\text { Reg Lordelo } \\
\text { Reg Montalegre } \\
\text { Reg Gimonde }\end{array}$ & $\begin{array}{l}0,14 \\
0,05 \\
0,06 \\
0,06\end{array}$ & $\begin{array}{l}-0,23 \\
-0,20 \\
-0,22 \\
-0,21\end{array}$ & $\begin{array}{l}0,07 \\
0,12 \\
0,06 \\
0,14\end{array}$ & $\begin{array}{l}-0,37 \\
-0,41 \\
-0,30 \\
-0,33\end{array}$ & $\begin{array}{l}-0,34 \\
-0,27 \\
-0,33 \\
-0,31\end{array}$ & $\begin{array}{l}-0,31 \\
-0,34 \\
-0,43 \\
-0,28\end{array}$ & $\begin{array}{l}-0,17 \\
-0,07 \\
-0,14 \\
-0,10\end{array}$ & $\begin{array}{r}0,11 \\
0,21 \\
0,21 \\
-0,05\end{array}$ & $\begin{array}{l}0,10 \\
0,07 \\
0,04 \\
0,07\end{array}$ & $\begin{array}{l}-0,21 \\
-0,18 \\
-0,19 \\
-0,21\end{array}$ \\
\hline 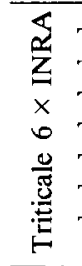 & $\begin{array}{l}\text { Trit. } 532-43 \\
\text { Trit. } 533-A-10 \\
\text { Trit. } 532-6 \\
\text { Trit. } 532-21 \\
\text { Trit. } 532-15 \\
\text { Trit. } 532-9 \\
\text { Trit. } 532-4\end{array}$ & $\begin{array}{l}0,25 \\
0,11 \\
0,19 \\
0,28 \\
0,30 \\
0,22 \\
0,37\end{array}$ & $\begin{array}{l}0,29 \\
0,18 \\
0,22 \\
0,31 \\
0,33 \\
0,29 \\
0,37\end{array}$ & $\begin{array}{l}0,24 \\
0,10 \\
0,26 \\
0,24 \\
0,29 \\
0,26 \\
0,27\end{array}$ & $\begin{array}{l}0,31 \\
0,17 \\
0,21 \\
0,19 \\
0,26 \\
0,23 \\
0,30\end{array}$ & $\begin{array}{l}0,21 \\
0,36 \\
0,20 \\
0,19 \\
0,11 \\
0,15 \\
0,18\end{array}$ & $\begin{array}{l}0,13 \\
0,33 \\
0,19 \\
0,09 \\
0,08 \\
0,16 \\
0,06\end{array}$ & $\begin{array}{l}-0,20 \\
-0,10 \\
-0,15 \\
-0,20 \\
-0,21 \\
-0,17 \\
-0,25\end{array}$ & $\begin{array}{l}-0,19 \\
-0,18 \\
-0,34 \\
-0,29 \\
-0,24 \\
-0,22 \\
-0,16\end{array}$ & $\begin{array}{l}0,23 \\
0,15 \\
0,20 \\
0,26 \\
0,27 \\
0,23 \\
0,32\end{array}$ & $\begin{array}{l}0,28 \\
0,21 \\
0,18 \\
0,24 \\
0,27 \\
0,28 \\
0,34\end{array}$ \\
\hline 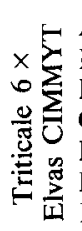 & 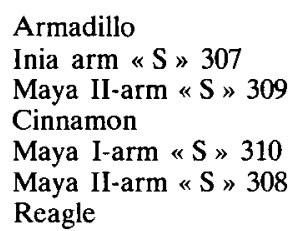 & $\begin{array}{l}-0,29 \\
-0,28 \\
-0,25 \\
-0,30 \\
-0,26 \\
-0,11 \\
-0,07\end{array}$ & $\begin{array}{r}-0,20 \\
-0,20 \\
-0,16 \\
-0,20 \\
-0,14 \\
0,05 \\
0,06\end{array}$ & $\begin{array}{l}-0,18 \\
-0,18 \\
-0,26 \\
-0,17 \\
-0,20 \\
-0,17 \\
-0,10\end{array}$ & $\begin{array}{r}-0,17 \\
-0,02 \\
-0,09 \\
-0,07 \\
-0,12 \\
0,03 \\
0,09\end{array}$ & $\begin{array}{r}-0,05 \\
-0,07 \\
0,02 \\
-0,10 \\
-0,15 \\
0,07 \\
0,19\end{array}$ & $\begin{array}{r}-0,06 \\
-0,06 \\
0,06 \\
0,02 \\
0,09 \\
0,27 \\
0,22\end{array}$ & $\begin{array}{l}0,42 \\
0,26 \\
0,27 \\
0,45 \\
0,29 \\
0,18 \\
0,16\end{array}$ & $\begin{array}{l}0,32 \\
0,31 \\
0,16 \\
0,25 \\
0,22 \\
0,16 \\
0,10\end{array}$ & $\begin{array}{c}-0,28 \\
-0,30 \\
-0,24 \\
-0,31 \\
-0,25 \\
-0,002 \\
0,05\end{array}$ & $\begin{array}{r}-0,23 \\
-0,22 \\
-0,15 \\
-0,22 \\
-0,13 \\
0,23 \\
0,19\end{array}$ \\
\hline
\end{tabular}

Pour chacun des 21 cultivars intervenant dans l'expérimentation, le tableau 2 rapporte les valeurs des paramètres $1_{\mathrm{i}}$ (effet principal génotype) et $\mathrm{u}_{1 \mathrm{i}}$ (facteur « génotype » participant au terme multiplicatif de rang 1 structurant l'interaction). On voit que, dans de très nombreux cas, n'apparaît pas la relation $u_{1 i}= \pm 1_{i}$, qui traduit l'existence d'une relation linéaire entre les $Y_{\mathrm{ij}}$ et les effets « lignes » et « colonnes». En fait, les déviations par rapport à cette relation sont beaucoup plus fréquentes dans les groupes des blés et des seigles que dans les deux groupes triticale.

Pour les productions de grain, de paille, de protéines, chacun des 4 groupes de génotypes se comporte de façon relativement homogène; les lignées de triticale INRA présentent des valeurs positives pour les effets principaux $1_{i}$ et les facteurs $u_{1 \mathrm{i}}$; les seigles régionaux présentent des valeurs positives pour les effets principaux, négatives pour les paramètres d'interaction; les 2 autres groupes présentent des valeurs plutôt négatives ou voisines de 0 , à l'exception de « Maya II-Arm S 308 » et de « Beagle » pour lesquels les contributions aux interactions sont positives. Dans un système d'axes $\left(1_{i}, u_{1 i}\right)$, les triticales INRA sont dans le quadrant 1 , les seigles dans le quadrant 2 , les triticales CIMMYT et les blés dans le quadrant 3.

Pour ce qui concerne les teneurs en protéines, 2 groupes présentent des valeurs globalement négatives au niveau de l'effet principal $1_{i}$ et de la contribution $u_{i}$ à l'interaction : les 
lignées triticale INRA et les cultivars de blé ; les seigles présentent des effets principaux négatifs, mais des contributions aux interactions généralement positives; les lignées CIMMYT offrent des valeurs globalement positives.

Pour le poids de 1000 grains, les lignées INRA de triticale et les seigles présentent des valeurs opposées: positives pour les unes (quadrant 1 dans le système d'axes $1_{i}$, $\mathrm{u}_{\mathrm{i}}$ ), négatives pour les autres. A l'intérieur de chacun des 2 autres groupes, les comportements ne sont pas homogènes : on trouve des effets principaux positifs associés à des contributions aux interactions négatives ( Barbela ») ou positives («Beagle $)$ ), comme des effets principaux négatifs associés à des contributions négatives ("Armadillo ») ou positives («Maya I-Arm S 310»: quadrant 4 dans un système d'axes $1_{\mathrm{i}}, \mathrm{u}_{1 \mathrm{i}}$ ).

Pour schématiser à l'extrême ces résultats, on peut dire que :

- le groupe des triticales CIMMYT et le groupe des blés ont des comportements généraux assez voisins; le critère qui les différencie le plus nettement est celui de la «teneur en protéines»;

- le groupe des lignées INRA de triticale présente un ensemble de valeurs pour les paramètres $1_{i}$ qui l'apparente au groupe des seigles régionaux; les 2 groupes se différencient nettement au niveau du caractère "poids de 1000 grains » et au niveau des paramètres d'interaction caractérisant les productions qui prennent des valeurs opposées.

La signification biologique que l'on peut attribuer à ces caractéristiques sera discutée plus loin.

\section{B. Résultats de l'analyse en composantes principales (ACP)}

Le pourcentage d'inertie des premiers axes sont les suivants :

axe $1: 58$ p. 100 ,

axe $2: 23$ p. 100 ,

axe 3 : 9 p. 100 .

L'axe 4 a un taux d'inertie inférieur à celui d'un caractère unique (4 p. 100).

L'axe 1 est fortement corrélé positivement avec les variables: productions de grain (PG), productions de protéines (PP), productions de paille (PA), et négativement avec la variable : teneur en protéines (TP) (tabl. 3). Il souligne donc l'antagonisme entre le paramètre «teneur» et les paramètres de production. L'axe 2 se définit essentiellement en fonction du paramètre : poids de 1000 grains (MG), auquel il est corrélé négativement. L'axe 3 est corrélé positivement avec la teneur en protéines et la production de paille.

Dans le systène d'axes 1-2 (p. 100 d'inertie cumulée : $81,3 \mathrm{HS}$ ) se différencient nettement 3 groupes de génotypes (fig. 1): les seigles, d'une part, dans le quadrant 1, qui se définissent en opposition aux facteurs «poids de 1000 grains " et «teneur en protéines » et dans une position favorable par rapport aux facteurs de production; les triticales INRA, d'autre part, dans le quadrant 2, en conjonction avec des facteurs de production et un poids de 1000 grains élevés; l'ensemble des génotypes CIMMYT, enfin, groupés av. pôle négatif de l'axe 2, dans la direction des teneurs en protéines élevées mais des productions faibles.

On remarque aussi, d'une part. que les blés «Siete Cerros » et «Autonomia » sont très proches de ce groupe de génotypes, alors que la variété « Barbela » se rattache au noyau INRA; d'autre part, que les lignées « Maya II-

\section{TABLEAU 3}

Analyse en composantes principales: coefficients de corrélation axes/variables. Principal component analysis: correlation coefficients for axes/variables.

\begin{tabular}{|c|c|c|c|}
\hline & Axe 1 & Axe 2 & Axe 3 \\
\hline MG 2 & 0,11 & $-0,96$ & 0,11 \\
\hline MG 3 & 0,47 & $-0,80$ & 0,19 \\
\hline MGC & 0,47 & $-0,86$ & $-0,05$ \\
\hline MGM & 0,28 & $-0,80$ & 0,41 \\
\hline MGB & 0,28 & $-0,86$ & 0,28 \\
\hline PG 2 & 0,81 & $-0,46$ & $-0,10$ \\
\hline PG 3 & 0,99 & $-0,01$ & 0,03 \\
\hline $\mathrm{PGC}$ & 0,94 & 0,07 & $-0,20$ \\
\hline PGM & 0,87 & 0,34 & 0,16 \\
\hline PGB & 0,89 & 0,34 & 0,15 \\
\hline PA 2 & 0,63 & $-0,69$ & $-0,10$ \\
\hline PA 3 & 0,83 & 0,10 & 0,44 \\
\hline PAC & 0,70 & 0,61 & 0,18 \\
\hline PAM & 0,74 & 0,35 & 0,40 \\
\hline PAB & 0,64 & 0,38 & 0,51 \\
\hline TP 2 & $-0,83$ & 0,17 & 0,51 \\
\hline TP 3 & $-0,87$ & $-0,008$ & 0,41 \\
\hline TPC & $-0,84$ & $-0,07$ & 0,49 \\
\hline TPM & $-0,78$ & $-0,19$ & 0,51 \\
\hline ТРВ & $-0,89$ & $-0,17$ & 0,30 \\
\hline PP 2 & 0,76 & $-0,50$ & $-0,02$ \\
\hline PP 3 & 0,98 & $-0,04$ & 0,11 \\
\hline PPC & 0,94 & 0,09 & $-0,15$ \\
\hline PPM & 0,80 & 0,30 & 0,38 \\
\hline PPB & 0,85 & 0,33 & 0,28 \\
\hline $\begin{array}{l}\text { Taux d'inertie } \\
(\%)\end{array}$ & 58 & 23,2 & 9,3 \\
\hline
\end{tabular}

MG: poids de 1000 grains.

PG : production de grain.

PA: production de paille.

TP : teneurs en protéines.

$\mathrm{PP}$ : production de protéines.

$2,3, \mathrm{C}, \mathrm{M}, \mathrm{B}$ : lieux d'expérimentation.

Armadillo S 308 » et « Beagle » se différencient légèrement des autres lignées CIMMYT.

Dans le système d'axe 1-3 (p. 100 d'inertie cumulée : 67,3 HS), les génotypes se différencient en 2 grands groupes caractérisés par leur aptitude ou leur inaptitude à fournir des productions élevées (2): la discrimination se fait essentiellement sur l'axe 1. Le pouvoir discriminant de l'axe 3 est faible et permet seulement de sortir du nuage "CIMMYT » les blés « Autonomia " et "Siete Cerros » qui présentent des teneurs en protéines plus faibles.

\section{DISCUSSION}

\section{A. Interprétation des résultats de l'analyse de MANDEL}

Si l'on essaie de définir la signification biologique $\mathrm{B}$ des différents paramètres dégagés par ce type d'analyse, celle de l'« effet principal " $1_{i}$ est assez claire. Les valeurs obtenues sont un reflet de la valeur moyenne du génotype pour le caractère étudié, affectées du coefficient « $L$ » pour laquelle les valeurs calculées sont généralement plus élevées que celles obtenues pour le coefficient $\mathrm{C}$ de l'effet principal " milieu».

Celle de la contribution du génotype au $1^{\text {er }}$ terme multiplicatif structurant l'interaction $\left(\mathrm{u}_{\mathrm{i}}\right)$ est un peu moins 


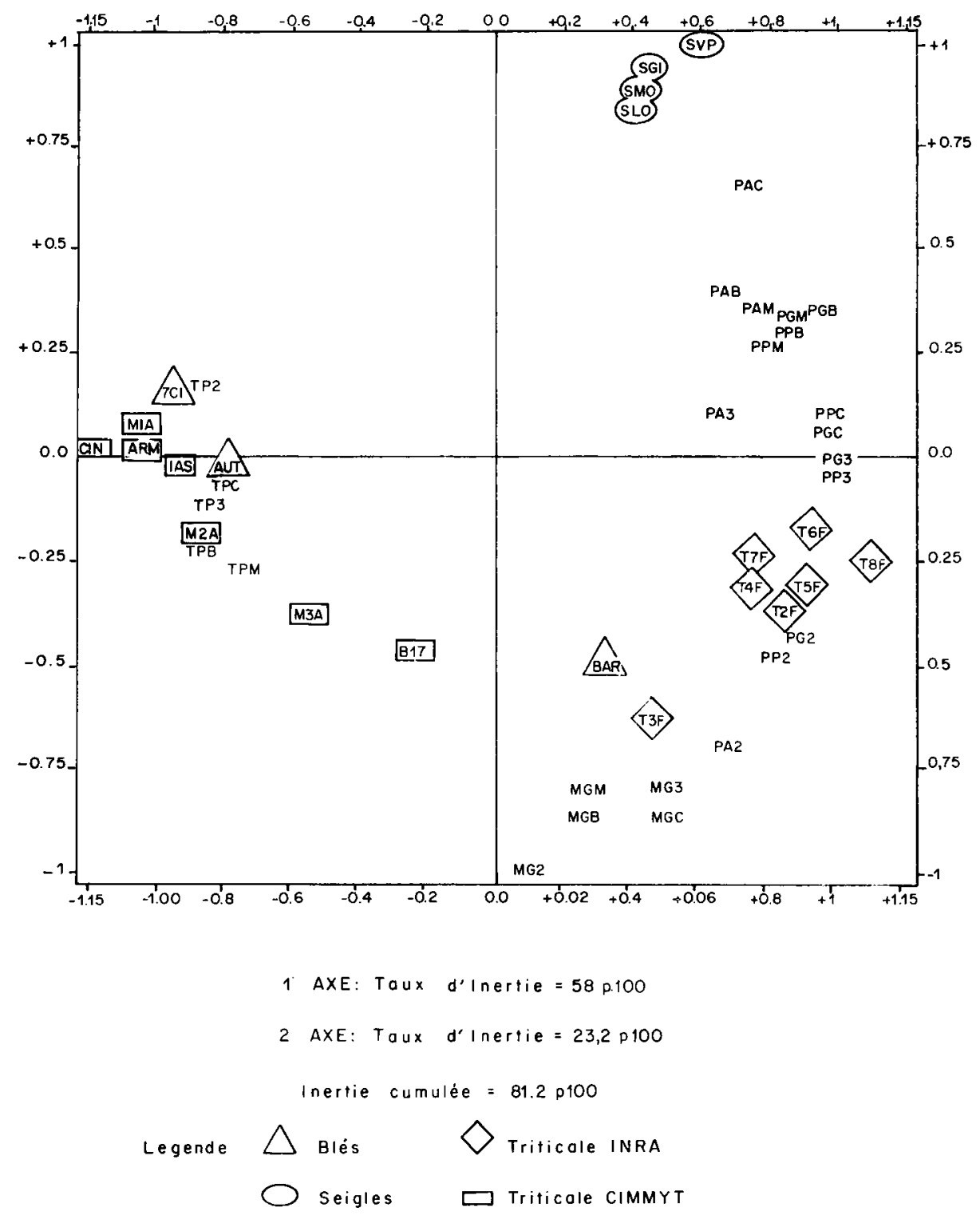

Figure 1

Représentation sur le plan des axes 1, 2 des génotypes dans l'analyse en composantes principales.

facile à cerner. Pour ce qui concerne la valeur absolue de cette contribution, on peut admettre qu'elle reflète la sensibilité du génotype aux variations du milieu ; quant au signe, il se définit par rapport aux signes des contributions aux interactions des différents milieux. Dans le cas présent, les milieux «pauvres » (TT3, M, B) présentent régulièrement des contributions négatives, Vila Real (TT2) des contributions positives, Carlão des contributions tantôt positives (production de grain), tantôt négatives (production de protéines). Par conséquent, la contribution génotypique sera positive pour des génotypes interagissant favorablement en milieux riches, négative pour ceux répondant mal en milieux riches mais favorablement en milieux pauvres. Suivant la production «espérée » en tel ou tel milieu, on fera appel à l'une ou l'autre de ces catégories de génotypes: les lignées INRA de triticale sont plus intéressantes à exploiter dans les «meilleurs " milieux, les seigles régionaux dans les situations pauvres. On peut schématiser leurs réactions en disant que leurs performances respectives dans leurs milieux de prédilection sont meilleures qu'attendu selon une relation linéaire.
Representation on the diagram of axes 1 and 2 of the genotypes in the principal component analysis.

Les représentations graphiques des matrices des produits $\left(\mu_{1 \mathrm{i}} \mathrm{v}_{1 \mathrm{j}}\right)$ (fig. $\left.3,4,5,6,7\right)$ permettent de visualiser ces résultats. On voit que, pour les paramètres de production (grain : fig 3, paille : fig. 4, protéines : fig. 5), les génotypes INRA réagissent de façon homogène, régulière et en opposition avec les autres cultivars; ils se caractérisent par un maximum dans le milieu le plus productif, TT2, et une dépression relative à Bragança et Montalegre. Les blés et les lignées triticale CIMMYT se comportent de façon assez semblable ; ils ne répondent pas favorablement en TT2, et ont un comportement relativement plus satisfaisant en TT3, TTM et TTB. Les seigles semblent très pénalisés en TT2, principalement pour la production de paille. On se rend compte également que 2 génotypes de triticale CIMMYT ont tendance à se comporter de façon assez semblable aux génotypes INRA ( « Maya II-Arm S 308 » et « Beagle», $\mathrm{n}^{\circ} 20$ et 21 des figures).

$\mathrm{Au}$ niveau des interactions concernant le poids de 1000 grains, on voit que les seigles se distinguent des autres groupes par une interaction très nettement défavorable en TT2 (fig. 6). D'autre part, le groupe des lignées INRA se 


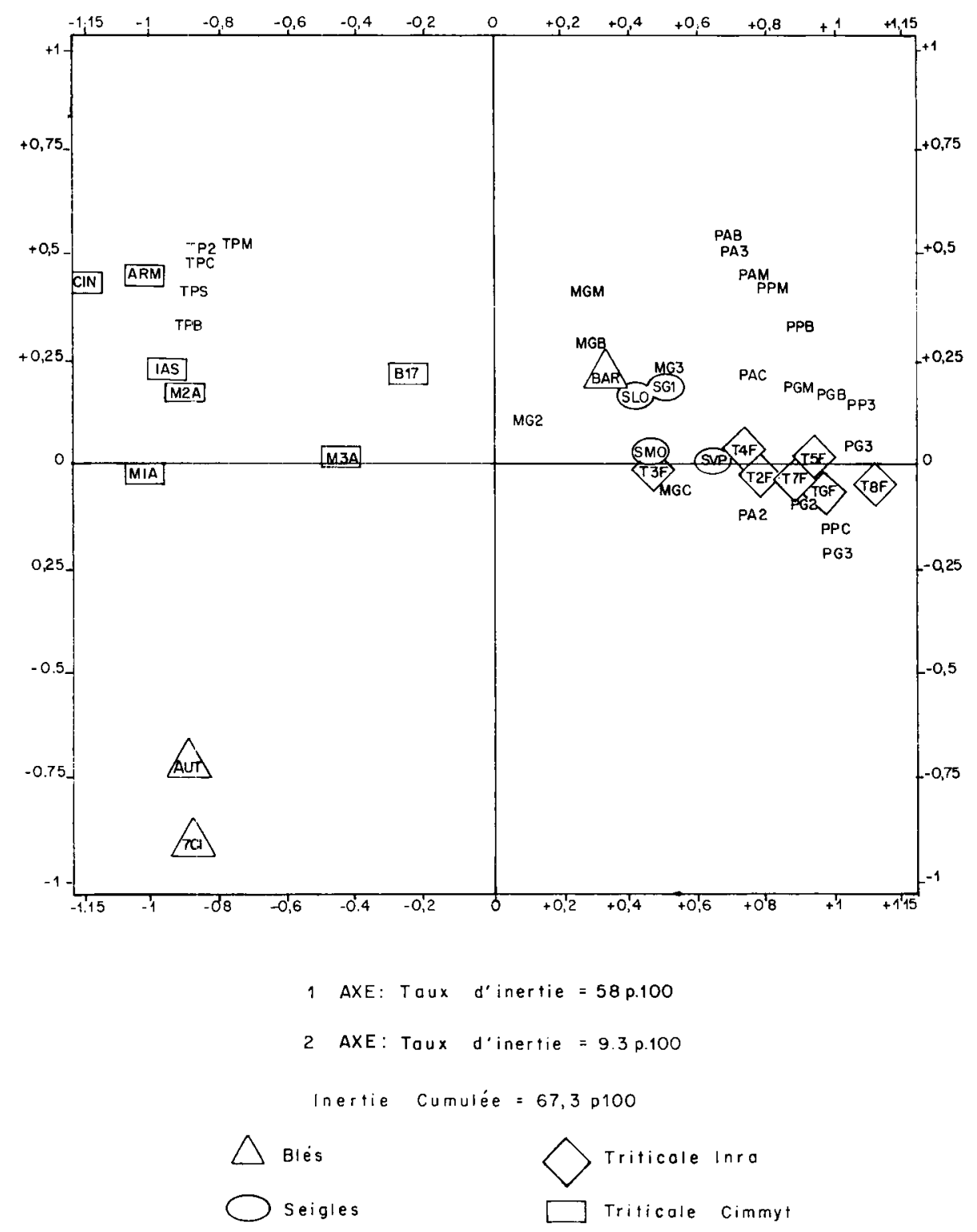

Figure 2

Représentation sur le plan des axes 1, 3 des génotypes dans l'analyse en composantes principales.

Representation on the diagram of axes 1, 3 of the genotypes in the principal component analysis.

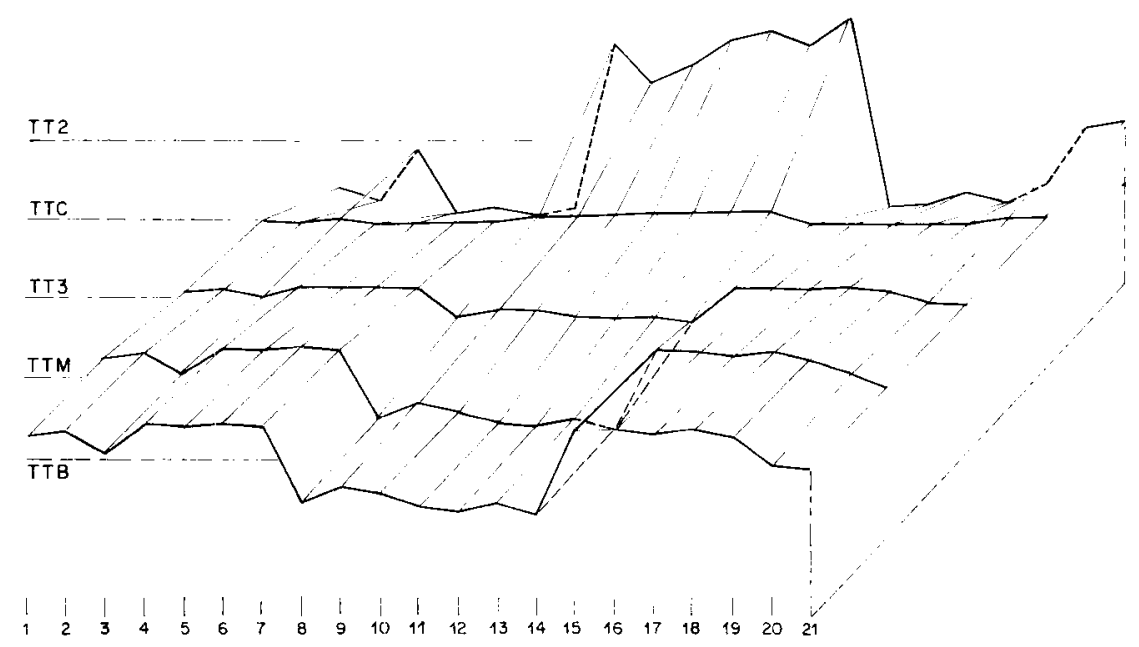

Figure 3

Représentation graphique des produits $\left(u_{i} v_{j}\right)$ de rang 1 structurant l'interaction, pour la production de grain. the interaction for grain production. 


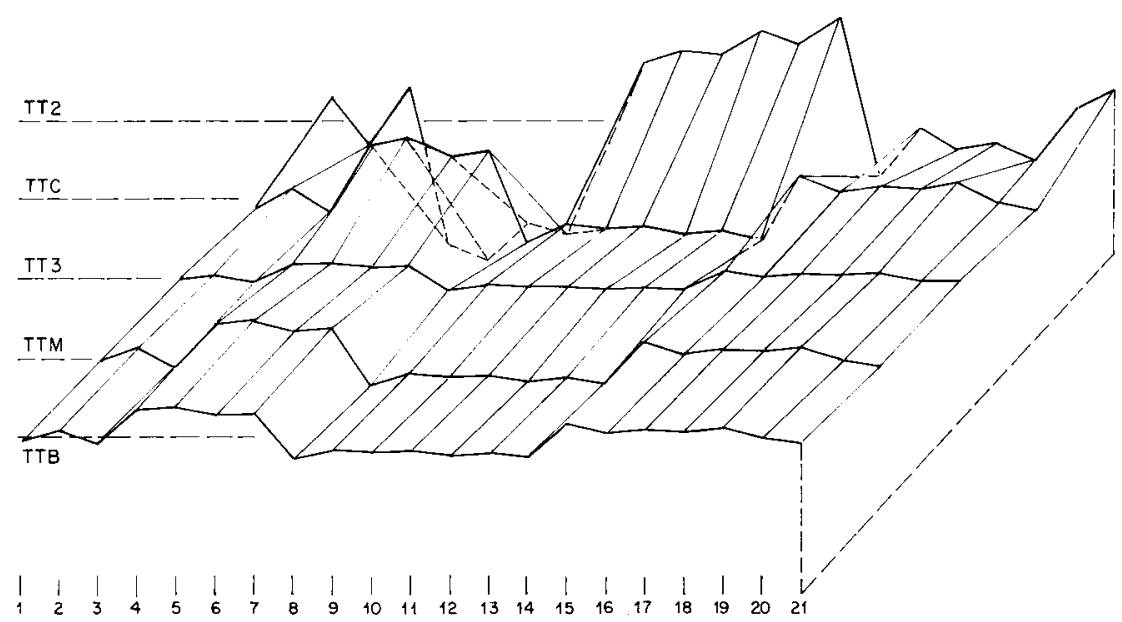

Figure 4

Représentation graphique des produits $\left(u_{i} v_{j}\right)$ de rang 1 , pour la production de paille.

Graphic representation of the first-level products $\left(u_{i} v_{j}\right)$ structuring the interaction for straw production.

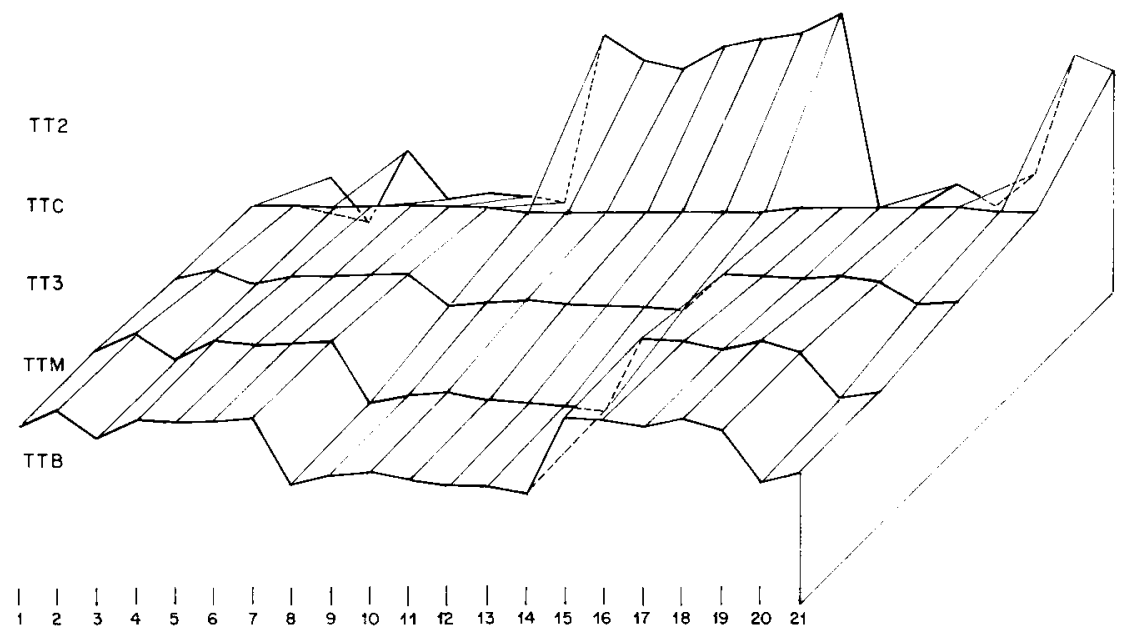

Figure 5

Représentation graphique des produits $\left(u_{i} v_{j}\right)$ de rang 1 , pour la production de protéines.

Graphic representation of the first-level products $\left(u_{i} v_{j}\right)$ structuring the interaction for protein production.

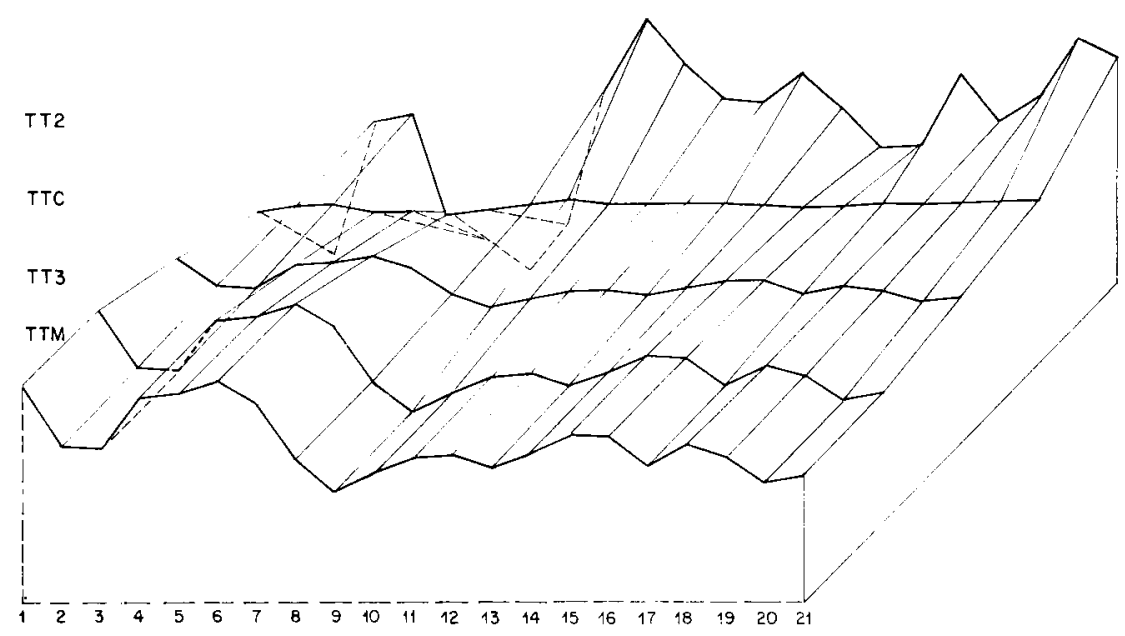

Figure 6

Représentation graphique des produits $\left(u_{i} v_{j}\right)$ de rang 1 , pour le poids de 1000 grains.
Graphic representation of the first-level products $\left(u_{i} v_{j}\right)$ structuring the interaction for 1000 kernel weight. 


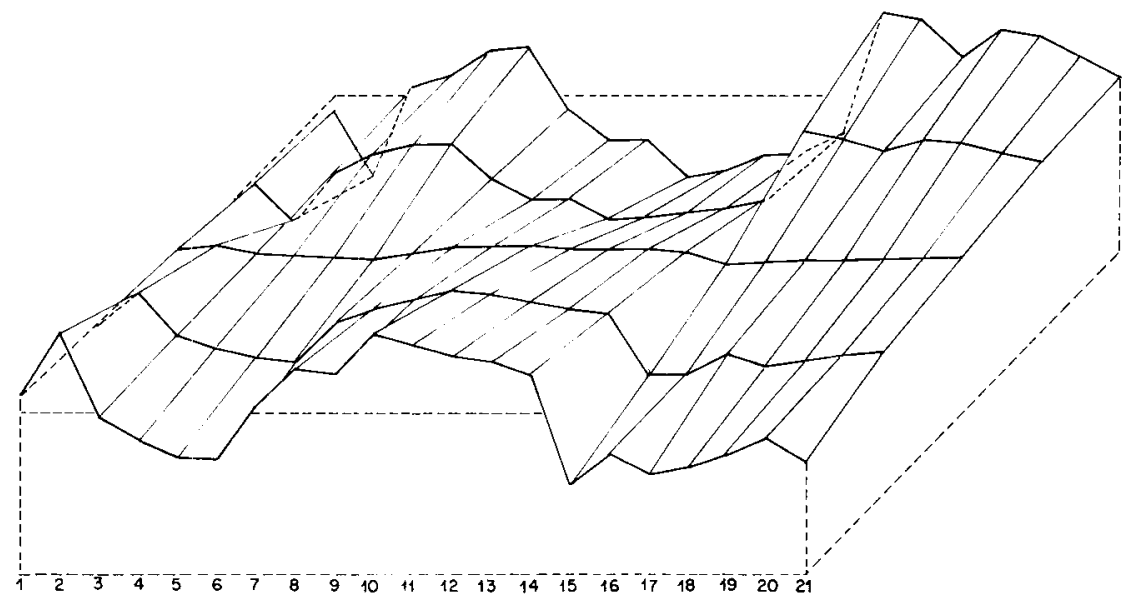

Figure 7

Représentation graphique des produits $\left(u_{i} v_{j}\right)$ de rang 1 , pour la teneur en protéines.

comporte différemment des autres groupes pour ce qui est des teneurs en protéines (fig. 7).

\section{Influence du nombre de milieux pris en compte}

Les valeurs obtenues pour les différents paramètres « effet principal génotype » et « contribution du génotype à l'interaction" dépendent fortement de la qualité et du nombre des partenaires entrant dans l'analyse, qu'il s'agisse des génotypes ou des milieux.

Nous avons cherché en particulier à voir comment évolue «l'effet génotype " $\mathrm{l}_{\mathrm{i}}$ quand l'on modifie le nombre et la «qualité » des milieux d'expérimentation pour le caractère «rendement en grain ». Nous avons progressivement soustrait à l'analyse les 3 milieux plus « riches", puis les 3 plus "pauvres». Les valeurs correspondantes de l'effet génotype pour chacun des 21 cultivars étudiés sont représentés graphiquement au tableau 4.

Si l'on élimine de l'analyse les milieux les plus fertiles, on peut constater 3 types de réactions:

- une stabilité de l'évaluation de l'effet génotype (cas des triticales CIMMYT),

- une diminution de la valeur de cet effet: cas des triticales INRA, du blé "Siete Cerros»,

- une augmentation de la valeur de cet effet : cas des seigles régionaux et du blé «Barbela».

Plus les milieux retenus dans l'analyse sont des milieux "pauvres», plus le comportement des seigles apparait intéressant, au détriment de celui des triticales INRA.

Par contre, l'élimination des milieux pauvres de l'analyse influe assez peu sur les valeurs de l'effet principal génotype pour les lignées INRA et les lignées CIMMYT; au contraire, les valeurs obtenues pour les seigles locaux et pour le blé « Barbela » chutent assez sensiblement : il s'agit à l'évidence des cultivars pénalisés en milieux riches.

\section{B. Interprétation des résultats de l'A.C.P.}

Par rapport à l'analyse précédente, ce type d'analyse a l'avantage de permettre une meilleure visualisation des liaisons, d'une part, entre les caractères, d'autre part, entre génotypes. Il a cependant l'inconvénient de ne pas permettre de différencier nettement l'effet principal " génotype " de l'interaction génotype $\times$ milieu, qui devient difficile à
Graphic representation of the first-level products $\left(u_{i} v_{j}\right)$ structuring the interaction for protein content.

appréhender même par une analyse fine des positions respectives des génotypes et des milieux.

Les conclusions que l'on pourra tirer quant à l'adéquation d'un génotype à un milieu donné seront donc moins précises que dans le cas précédent. Ceci est dû en partie au fait que le nombre de variables étudiées est faible et que, de surcroît, ces dernières sont corrélées entre elles. Néanmoins, cette analyse permet de dégager les mêmes grandes tendances que l'analyse de MANDEL.

Dans le système d'axes définis par les caractères pris en compte, 4 groupes de génotypes se différencient facilement: le groupe des populations de seigle, le groupe des triticales INRA, le groupe des triticales CIMMYT, les 2 variétés de blé «Autonomia » et «Siete Cerros». Trois génotypes ne se rattachent directement à aucun de ces groupes : la variété "Barbela " et les lignées CIMMYT de triticale « Maya II-Arm S n ${ }^{0} 308$ » et « Beagle», dont les positions sont équidistantes des pôles CIMMYT, INRA et "blés » et très éloignés du nuage "seigle ».

Les nuages «seigle » et «triticale INRA », qui sont en corrélation avec les productions élevées et les faibles teneurs en protéines, se différencient nettement suivant l'axe 2, défini par le poids de 1000 grains, dont les valeurs sont faibles pour les seigles, élevées pour les triticales et « Barbela ». Les groupes « triticale CIMMYT » et « Autonomia-Siete Cerros» se différencient suivant l'axe 3, en corrélation avec la teneur en protéines et la production de paille.

Un examen plus poussé révèle que le groupe des triticales INRA est fortement corrélé avec les valeurs de production observées dans l'essai «Vila Real 2 », tandis que le groupe des seigles est plutôt corrélé avec les productions observées à Bragança, Montalegre et dans une moindre mesure à Carlão.

Cette analyse permet d'autre part de vérifier, au-delà de la visualisation des 3 grands groupes génotypiques définis précédemment, que la production de protéines dépend davantage de la production de matière sèche que de la teneur en protéines. Compte tenu de la liaison négative entre ces 2 composantes $(r=-0,85)$, il semble bien difficile d'améliorer simultanément les deux et, à court terme, une augmentation de la première se révèlera plus intéressante qu'une augmentation de la seconde pour une augmentation globale de la production de protéines (JESTIN, 1974 ; BERNARD et al., 1979). 


\section{TABLEAU 4}

Evolution des valeurs prises par l'effet génotype $l_{i}$ en fonction du nombre de milieux pris en compte dans l'analyse de Mandel pour le caractère

"rendement en grain»;

- élimination des milieux les plus fertiles,

- élimination des milieux les plus pauvres.

Variation of the values of the genotype effect $l_{i}$ according to the number of locations involved in the Mandel analysis for the character "grain production";

- elimination of the most fertile locations,

- elimination of the poorest locations.
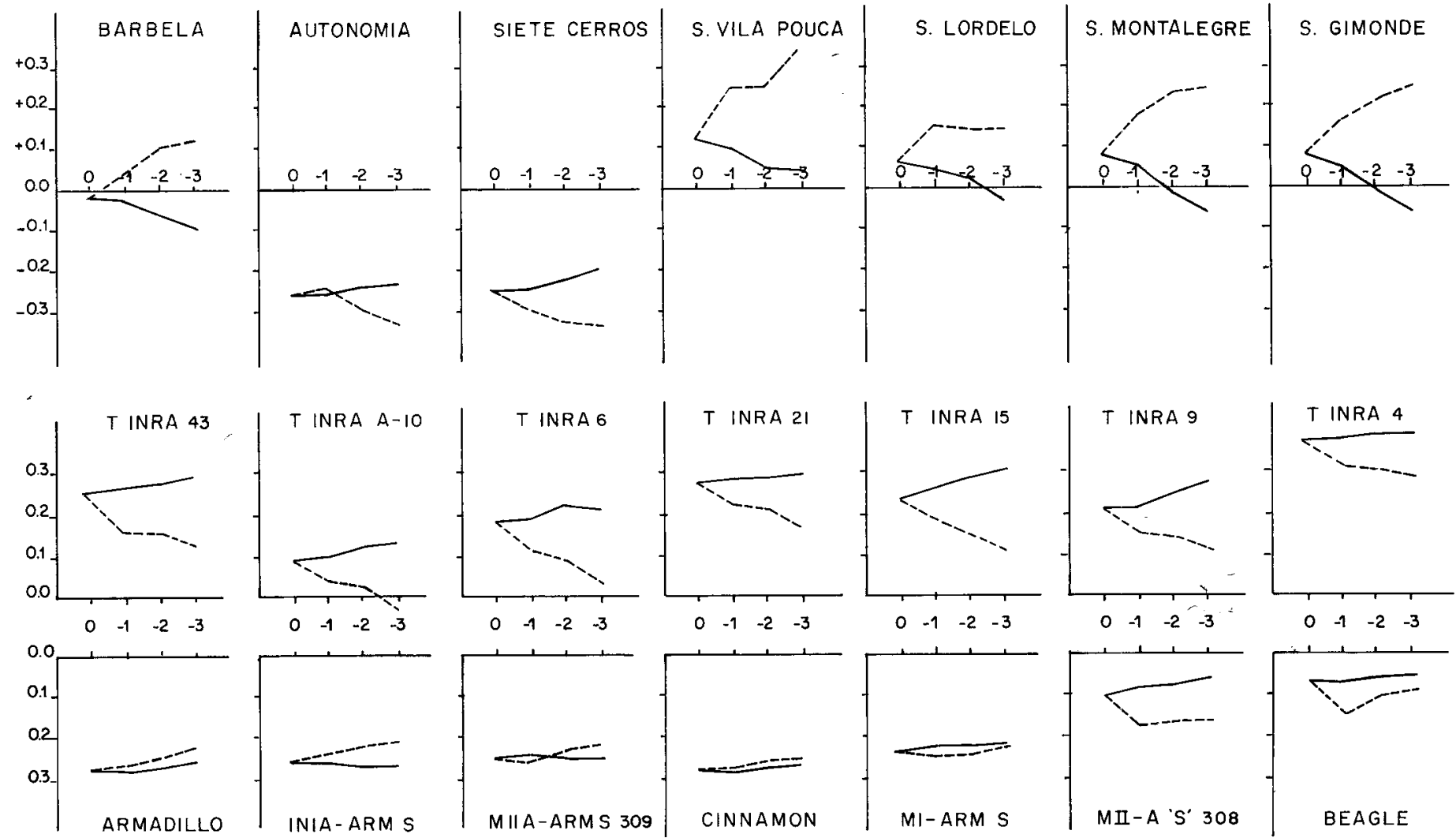

\section{CONCLUSIONS}

L'analyse d'une expérimentation multilocale suivant le modèle de MANDEL fournit des renseignements complémentaires de ceux obtenus par l'analyse des régressions des productions individuelles aux productions moyennes par milieu (tabl. 5 et fig. 1 de l'article précédent) (WRIGHT, 1976).

Il permet en effet de définir, en fonction d'un ensemble de milieux et d'une collection de génotypes, 2 caractéristiques différentes pour un génotype donné :

- son potentiel moyen, ou encore son «aptitude générale à la production », que l'on peut estimer par le signe et la valeur de la variable «effet du génotype »;

- son potentiel de réponse spécifique au milieu, ou encore son potentiel d'«aptitude spécifique à la production » dans un milieu donné, que l'on peut estimer par le signe et la valeur de la contribution de ce génotype à l'interaction "génotype $\times$ milieu ".

La définition de ces 2 caractéristiques peut être obtenue pour chaque génotype pour les différents paramètres de production susceptibles d'intéresser l'expérimentateur ou le sélectionneur. L'analyse de MANDEL ne permet cependant pas de définir les liaisons existant entre les différents paramètres qui doivent être établies par d'autres techniques.

Par contre, elle permet de dégager les similitudes ou les divergences de comportement entre les génotypes entrant dans l'analyse. Par exemple, au niveau des potentiels moyens de production, on retrouve la stratification en groupes que nous avions mentionnée dans la précédente publication. De plus, les convergences ou divergences de comportement apparaissent aussi très clairement au niveau des potentiels de réponse spécifique au milieu ; une illustration en est donnée par la comparaison des profils de réponse de chaque variété par rapport à la gamme de milieux (fig. 3 à 7). Deux groupes s'opposent alors très nettement : celui des populations locales de seigle, et celui des triticales INRA, alors que leurs "potentiels moyens" sont très proches. Le $1^{\text {er }}$ groupe interagit très favorablement avec les milieux de Bragança, Montalegre et Vila Real 3, le $2^{\mathrm{e}}$ avec les milieux «ûriches" de Carlấo et Vila Real 2. 
Les variétés de blé et les lignées de triticale du CIMMYT présentent des comportements plus hétérogènes et intermédiaires entre les 2 groupes précédents, tantôt semblables à un groupe, tantôt semblables à l'autre. Il convient en particulier de remarquer le comportement de la variété de blé «Barbela » qui, pour plusieurs paramètres, présente un profil d'interaction proche de celui des seigles. Les triticales CIMMYT présentent des profils voisins des seigles pour les productions de grain et de protéines, la teneur en protéines, mais semblables aux triticales INRA pour le poids de 1000 grains. Dans ce groupe des génotypes CIMMYT, les profils les plus semblables à ceux des lignées INRA sont obtenus régulièrement avec "Maya II-Arm S $n^{\circ} 308$ " et «Beagle». Les autres ont un comportement plus voisin des blés «Autonomia» et «Siete Cerros».

Contrairement à ce que l'on pouvait attendre du simple fait de leur identité génomique, ce sont donc les seigles et un groupe de triticales qui présentent les comportements de spécialisation les plus opposés, tandis que les blés et un autre groupe de triticales ont un comportement intermédiaire. Ces résultats sont contraires à ceux obtenus sur le même type d'essais réalisés en France (BERNARD \& GUEDES-PINTO, 1980) mais où les triticales INRA ont été confrontés à des variétés de blé et de seigle différentes de celles observées ici : ils présentent alors un profil de réponse intermédiaire entre ceux des représentants des espèces «parentes ».

Il semble que la caractéristique de réaction spécifique d'un génotype au milieu est fonction bien sûr de son patrimoine génétique, mais présente l'empreinte de l'environnement dans lequel ce génotype a été sélectionné. Dans un milieu donné, le sélectionneur ne peut que tenir compte du couple "génotype $\times$ milieu »: il ne peut dissocier l'effet principal génotype du paramètre de réponse spécifique du génotype au milieu. Autrement dit, tout génotype sélectionné l'est en fonction de son adaptation au milieu de sélection. On peut supposer que ce génotype présentera généralement une interaction positive dans les milieux de même type que le milieu de sélection et négative dans des milieux très différents. On aura donc intérêt à sélectionner dans un milieu représentatif du territoire à coloniser : ce choix préliminaire du milieu et sa définition, devraient constituer la $1^{\text {re }}$ étape d'une démarche de sélection végétale.

Un autre problème est l'amplitude de cette aptitude à l'interaction qui détermine l'écart entre la production espérée et la production observée. On peut supposer que l'environnement joue également un rôle, mais cette fois en raison de ses propres variations annuelles. Un milieu « très constant" sur la période de sélection (3 à 5 ans) doit favoriser l'émergence de génotypes très «pointus », très étroitement spécialisés à un type de conditions pédologiques et climatiques. Si au contraire l'environnement varie fortement d'une année à la suivante, le classement des génotypes ne sera pas le même et le choix plus difficile à faire, mais il est vraisemblable que les génotypes retenus auront une adaptation plus large. L'expérimentation sous forme d'essais multilocaux au cours de la phase de sélection doit remplir ce rôle. D'autre part, de telles études, conduites sur plusieurs génotypes, plusieurs lieux, plusieurs années, devraient permettre de définir les meilleurs milieux de sélection en fonction des objectifs recherchés (spécificité ou adaptation large). Il nous semble qu'une classification des milieux de sélection pourrait être faite en fonction de la valeur de leur effet principal et de la valeur de leur participation à l'interaction génotype $\times$ milieu selon le modèle suivant :

\begin{tabular}{|c|c|}
\hline \multirow[b]{2}{*}{$\begin{array}{l}\text { Effet } \\
\text { principal } \\
\text { « milieu » }\end{array}$} & $\begin{array}{l}\text { Participation du milieu } \\
\text { à l'interaction } \\
(G \times E)\end{array}$ \\
\hline & $\begin{array}{cc}\text { faible } & \text { forte } \\
\text { (en valeur } & \text { (en valeur } \\
\text { absolue) } & \text { absolue) }\end{array}$ \\
\hline élevé & $\begin{array}{ll}\text { milieu de diversifi- très forte produc- } \\
\text { cation, hautement } & \text { tion pour des géno- } \\
\text { "permissif " } & \text { types très spéciali- } \\
& \text { sés (« terroir") }\end{array}$ \\
\hline moyen & $\begin{array}{l}\text { bonne discrimina- favorable à la sélec- } \\
\text { tion des génotypes tion de génotypes } \\
\text { en fonction essen- hautement spécia- } \\
\text { tiellement de leur lisés } \\
\text { « effet principal» }\end{array}$ \\
\hline faible & $\begin{array}{ll}\text { sélection de génoty- milieu "dur », fa- } \\
\text { pes à très haute vorisant les génoty- } \\
\text { valeur de l'effet pes très spécialisés, } \\
\text { principal génotype très étroitement } \\
& \begin{array}{l}\text { adaptés }\end{array}\end{array}$ \\
\hline
\end{tabular}

D'autre part, dans l'optique d'une amélioration de la valeur adaptative moyenne d'un ensemble de génotypes, les paramètres "effet principal génotype " et "valeur du paramètre d'interaction " devraient être pris en compte au niveau du choix des parents entrant dans un croisement, au même titre que des caractéristiques plus ponctuelles, phénotypiquement plus aisément identifiables.

Sur le plan génétique, l'opposition entre les triticales INRA et les triticales CIMMYT peut sembler étonnante. En ACP, ils apparaissent équidistants des blés et des seigles, mais dans des zones opposées.

Dans ce contexte, la situation particulière de « Maya IIArm $S n^{\circ} 308$ » et surtout de « Beagle » apporte un élément de discussion intéressant. "Beagle » est, parmi les triticales du CIMMYT alors disponibles (1978), celui qui est phénotypiquement et agronomiquement le plus proche des génotypes INRA. On sait d'autre part que les triticales peuvent présenter, ou non, l'intégralité du génome du seigle, les éléments manquants de ce dernier étant remplacés par des chromosomes du génome D (BERNARD \& BERNARD, 1978). A cet égard, les lignées de triticale INRA entrant dans cette analyse sont "non substituées", alors que les lignées CIMMYT de type «Armadillo» présentent souvent plusieurs substitutions R/D (MERKER, 1975 ; GUSTAFSON et al., 1980). « Beagle » est aussi un triticale non substitué. La présence de l'intégralité du génome seigle confère d'ailleurs aux lignées de triticale concernées existant à cette époque (1978) une grande similitude phénotypique qui peut être due, ou non, à des relations de consanguinité. Le matériel français est en effet issu de croisements faisant intervenir un groupe de matériel américain (CAUDERON \& BERNARD, 1980) auquel «Beagle » est peut-être rattaché. D'autre part, le niveau des performances agronomiques est lié, pour un certain type de matériel, à la présence de l'intégralité du génome seigle (PILCH, 1981). Une confrontation plus complète entre les résultats agronomiques d'une collection de génotypes de préférence non consanguins, d'une part, et les fiches d'identité chromosomique de ces génotypes, d'autre part, pourrait permettre de déceler quels sont, parmi les éléments du génome de seigle, ceux qui ont le rôle le plus important dans la détermination des qualités agronomiques du triticale, tant au point de vue de la productivité que des facultés d'adaptation. Il est en effet possible que les corrélations actuellement observées soient liées à une 
cohésion fonctionnelle moins bonne dans les types substitués que dans les types non substitués, autrement dit, que les substitutions ne soient pas «optimales».

Cette démarche pourrait aussi permettre de proposer une voie d'approche pour l'étude physiologique des propriétés adaptatives qui devrait être le lien naturel entre le comportement au champ et la connaissance du génotype.

Reçu le 16 février 1982. Accepté le 29 mars 1983.

\section{REMERCIEMENTS}

Les auteurs remercient M. Gérard BRANLARD (INRA ClermontFerrand) pour les renseignements concernant l'utilisation des programmes d'analyse de MANDEL et d'analyse en composantes principales, ainsi que le Centro de Calculo Cientifico do Instituto Gulbenkian de Ciencias (Oeiras, Portugal) où les programmes ont été exécutés.

\section{RÉFÉRENCES BIBLIOGRAPHIQUES}

Anderson T. W., 1960. An introduction to multivariate statistical analysis. John Wiley \& Sons. inc. New York, London.

Benzecri J. P., 1973. L'analyse des données, T. 1, Dunod éd., Paris, $615 \mathrm{p}$.

Bernard M., Bernard S., 1978. Methods of gene transfer from bread wheat and rye to hexaploid triticale. Proc. 8th Eucarpia Congress "Interspecific Hybridization in Plant Breeding", Madrid, Spain, 1977, 181-189.

Bernard M., Branlard G., Farouk-El-Tallawi M., Laroche G. Bourdet A., Feillet P., 1979. Caractéristiques quantitatives et qualitatives des protéines de triticale : études de lignées sélectionnées en France. In : Seed Protein Improvement in Cereals and Grain Legumes. Proc. Int. Symposium, Neuherberg 4-8 September 1978, Vol. II, IAEA Vienne (1979), 237-259.

Bernard M., Guedes Pinto H., 1980. Analysis of the genotype environment interactions in triticale breeding. Hod. Rosl. Aklim. Nasien, 24 (5), 645-660.

Brian C., 1978. Interprétation statistique des essais « deux voies " : décomposition factorielle des résidus et étude de la structure des interactions. Ann. Amélior. Plant., 28 (4), 395-410.

Cauderon Y., Bernard M., 1980. Yield improvement from $(8 \mathrm{x} \times 6 \mathrm{x})$ crosses, and genic and cytoplasmic diversification in triticale. Hod. Rosl. Aklim. Nasien, 24 (4), 329-338.

Guedes Pinto H., Bernard M., 1983. Etude comparative de quelques cultivars de blé, seigle et triticale dans le nord du
Portugal. I. Productions de grain, de paille, de protéines. Agronomie, 3 (7).

Gustafson J., Bennett M. D., Larter E. N., 1980. Rye chromosome substitution and modification in 42-chromosome triticale. Hod. Rosl. Aklim. Nasien 24 (4), 373-382.

Johnson D. E., Graybill A., 1972. An analysis of a two-way model with interaction and no replication. J. am. Statist. Assoc., 67 (340), 862-868.

Jestin L., 1974. Les variations de la teneur en protéines brutes chez l'orge selon la variété et le milieu : étude préliminaire à partir d'essais culturaux. Ann. Amélior. Plant., 24 (4), 377-388.

Lefort P. L., 1977. L'interaction entre facteurs dans l'analyse de variance. Application à l'interaction génotype $\times$ milieu. Tocsin $d u$ Radiateur, 6, 444-453.

Mandel J., 1971. A new analysis of variance model for non-additive data. Technometrics, 13, 1-18.

Merker A., 1975. Chromosome composition of hexaploid triticale. Hereditas, 80, 41-52.

Pilch J., 1981. Rye chromosome constitution and the amount of telomeric heterochromatin of the widely and narrowly adapted CIMMYT hexaploid Triticales. Z. Pflanzenzüchtung, 87 (1), 56-68. Wright A. J., 1976. The significance for breeding of linear regression analysis of genotype-environment interactions. Heredity, 37 (1), 89-93. 